\title{
Stable recognition of TA interruptions by triplex forming oligonucleotides containing a novel nucleoside.
}

\section{SUPPORTING INFORMATION}

Yang Wang ${ }^{1}$, David A. Rusling ${ }^{2}$, Vicki E.C.Powers ${ }^{1}$, Oliver Lack ${ }^{1}$, Sadie D.

Osborne $^{1,3}$, Keith R. Fox ${ }^{2}$, Tom Brown ${ }^{1}$

${ }^{1}$ School of Chemistry, University of Southampton, Highfield, Southampton SO17 1BJ,

U.K.

${ }^{2}$ School of Biological Sciences, University of Southampton, Bassett Crescent East, Southampton SO16 7PX, U.K. 


\section{General chemical methods}

Reagents were purchased from Aldrich, Avocado, Cruachem, Fluka, Lancaster or Link Technologies Ltd. The following solvents were purified by distillation: methanol (over iodine and magnesium), THF (over sodium wire and benzophenone), DCM, DIPEA, pyridine and TEA (over calcium hydride). All chemical reactions were carried out under argon using oven dried glassware. Column chromatography was carried out under pressure using Fisher scientific DAVISIL 60A (35-70 micron) silica. Compounds were visualised by irradiation at $254 \mathrm{~nm}$ and/or by staining with anisaldehyde, potassium permanganate or phosphomolybdic acid (PMA). Thin layer chromatography was performed using Merck Kieselgel 60 F24 (0.22mm thickness, aluminium backed). ${ }^{1} \mathrm{H}$ NMR spectra were measured at $300 \mathrm{MHz}$ on a Bruker AC300 spectrometer or $400 \mathrm{MHz}$ on a Bruker DPX400 spectrometer and ${ }^{13} \mathrm{C}$ NMR spectra were measured at $75 \mathrm{MHz}$ and $100 \mathrm{MHz}$ on the same spectrometers. Chemical shifts are given in ppm relative to tetramethylsilane, and $J$ values are given in $\mathrm{Hz} .{ }^{31} \mathrm{P}$ NMR spectra were recorded on a Bruker AC300 spectrometer at $121 \mathrm{MHz}$ and were externally referenced to $85 \%$ phosphoric acid in $\mathrm{D}_{2} \mathrm{O}$. Low-resolution mass spectra were recorded using electrospray technique on a Fisons VG platform instrument in acetonitrile or a Waters ZMD quadrupole mass spectrometer in methanol and high-resolution mass spectra were recorded using electrospray on a Bruker APEX III FT-ICR mass spectrometer in methanol or acetonitrile. Infrared spectra were recorded on a Satellite FT-IR using a Golden Gate adapter and WIN FIRST-lite software. Absorptions are described as strong (s), medium (m), broad (b) or weak (w). Melting points were measured on a Gallenkamp electrothermal melting point apparatus and were uncorrected. 


\section{Synthesis of ${ }^{2 \mathrm{AE}} \mathrm{S}$ monomer 12}

\section{Isobutyl-3',5'-O-(1,1,3,3,-tetraisopropyldisiloxane-1,3-diyl)-D-ribose, 2}

Butan-2-ol $(100 \mathrm{~mL})$ was added to D-ribose $(10.0 \mathrm{~g}$, $66.7 \mathrm{mmol})$. The resulting suspension was stirred for 10 mins before adding a solution of acetyl chloride $\mathrm{mL})$ in butan-2-ol $(50 \mathrm{~mL})$. The mixture was left to stir at $\mathrm{rt}$ for $20 \mathrm{~h}$. Sodium bicarbonate $(5.0 \mathrm{~g})$ was added and the reaction mixture was filtered. The solvent was removed in vacuo to give a clear yellow oil. This was coevaporated with pyridine ( $3 \times 20$ $\mathrm{mL})$ and dried under high vacuum before redissolving in pyridine $(200 \mathrm{~mL})$. The solution was cooled to $-10{ }^{\circ} \mathrm{C}$ before adding 1,3-dichloro-1,1,3,3-tetraisopropyldisiloxane $(21.3 \mathrm{~mL}, 66.6 \mathrm{mmol})$ dropwise over $2 \mathrm{~h}$. The reaction mixture was then left to stir at $\mathrm{rt}$ for $4 \mathrm{~h}$. The solvent was removed in vacuo and the residue redissolved in DCM $(200 \mathrm{~mL})$. This was washed with water, $2 \mathrm{M} \mathrm{HCl}$, water again and finally brine. The solution was dried over sodium sulfate and the solvent removed in vacuo. The crude product was purified by column chromatography (9:1 hexane:ethyl acetate) to give $\mathbf{2}$ as an anomeric mixture as a colourless oil (22.2 g, 74\%).

$\mathrm{R}_{\mathrm{f}}\left(1: 1\right.$ hexane:ethyl acetate, $\left.\mathrm{B}^{\prime}\right)$ 0.38, 0.45; $v_{\max }($ neat $) / \mathrm{cm}^{-1} 3566(\mathrm{w}), 2944(\mathrm{~m}), 2867$ (m), $1464(\mathrm{~m}), 1385$ (w), 1323 (w), $1248(\mathrm{w}), 1136$ (w), 1066 (w), 1033 (s), 1006 (s), 991 $(\mathrm{s}), 883(\mathrm{~s}), 692(\mathrm{~s}) ; \delta_{\mathrm{H}}\left(400 \mathrm{MHz}, \mathrm{CDCl}_{3}\right)$ major anomer $(\alpha)$ : $5.05\left(1 \mathrm{H}, \mathrm{s}, \mathbf{H}^{1}\right), 4.50(1 \mathrm{H}$, $\left.\mathrm{dd}, J=10.5,5.0 \mathrm{~Hz}, \mathbf{H}^{3^{\prime}}\right), 3.97\left(1 \mathrm{H}, \mathrm{t}, J=5.0 \mathrm{~Hz}, \mathbf{H}^{2}\right), 3.81\left(1 \mathrm{H}\right.$, app.t, $\left.J=11.0 \mathrm{~Hz}, \mathbf{H}^{4^{\prime}}\right)$, $3.66\left(1 \mathrm{H}, \mathrm{dd}, J=11.0,6.0 \mathrm{~Hz}, \mathbf{H}^{5^{\prime}}\right), 3.60\left(1 \mathrm{H}, \mathrm{dd}, J=11.0,6.0 \mathrm{~Hz}, \mathbf{H}^{5^{\prime}}\right), 2.95(1 \mathrm{H}, \mathrm{bs}$, $s \mathrm{Bu}-\mathrm{CH}), 1.60\left(1 \mathrm{H}, \mathrm{bs}, \mathrm{OH}^{2}\right.$ ) $), 1.35-1.56$ (2H, m, $\left.s \mathrm{Bu}-\mathrm{CH}_{2}\right), 0.91-1.13$ (28H, m, TIPDS), 
0.85-0.89 $\left(6 \mathrm{H}, \mathrm{m}, s \mathrm{Bu}-\mathrm{CH}_{3}\right) ; \delta_{\mathrm{C}}\left(100 \mathrm{MHz}, \mathrm{CDCl}_{3}\right)$ major anomer $(\alpha): 103.7\left(\mathrm{~d}, \mathbf{C}^{\mathrm{l}}\right)$, $82.6\left(\mathrm{~d}, \mathbf{C}^{4}\right), 82.5\left(\mathrm{~d}, \mathbf{C}^{2}\right), 75.4\left(\mathrm{~d}, \mathbf{C}^{3}\right), 73.5(\mathrm{~d}, s \mathrm{Bu}-\mathbf{C H}), 64.9\left(\mathrm{t}, \mathbf{C}^{\mathbf{S}^{\prime}}\right), 30.3(\mathrm{~d}, s \mathrm{Bu}-$ $\mathbf{C H}_{2}$ ), 21.1 (q, sBu-CH$)_{3}$, 17.9, 17.9, 17.9, 17.8, 17.7, 17.6, 17.6 (q, TIPDS- $\mathrm{CH}_{3}$ ), 13.8, 13.8, 13.6, 13.3 (d, TIPDS-CH), 10.3 (q, sBu-CH$) ; m / z$ LRMS [ES $\left.{ }^{+}, \mathrm{MeOH}\right] 471$ $\left(\mathrm{M}+\mathrm{Na}^{+}, 100 \%\right) ; m / z$ HRMS $\left[\mathrm{ES}^{+}, \mathrm{MeOH}\right]$ found $471.2578\left(\mathrm{M}+\mathrm{Na}^{+}\right) \quad \mathrm{C}_{21} \mathrm{H}_{44} \mathrm{O}_{6} \mathrm{Si}_{2} \mathrm{Na}$ requires 471.2578 .

\section{Isobutyl-2'-O-ethyl-3',5'-O-(1,1,3,3,-tetraisopropyldisiloxane-1,3-diyl)-D-ribose, 4}

Compound 2 (21.4 g, $47.7 \mathrm{mmol})$ was dissolved in anhydrous DMF (20 mL) and cooled to $-10{ }^{\circ} \mathrm{C}$ (ice/methanol) before adding methyl bromoacetate $(11.3 \mathrm{~mL}, 119 \mathrm{mmol})$ followed by sodium hydride (60\% dispersion in mineral oil, $4.77 \mathrm{~g}, 119 \mathrm{mmol}$ ) portionwise. The reaction mixture was left to stir at $-10{ }^{\circ} \mathrm{C}$ for $1 \mathrm{~h}$ then $\mathrm{rt}$ for $3.5 \mathrm{~h}$. Saturated $\mathrm{KCl}$ solution $(200 \mathrm{~mL})$ was added and the reaction mixture extracted with diethyl ether. The organic layers were combined dried over sodium sulfate and the solvent removed in vacuo. The crude product was filtered through a silica plug (eluting with 9:1 hexane:ethyl acetate). The filtrate was condensed and the residue redissolved in THF (200 mL). Lithium borohydride $(1.99 \mathrm{~g}, 91.2 \mathrm{mmol})$ was added and the reaction mixture left to stir at $\mathrm{rt}$ for $3 \mathrm{~h}$. The reaction mixture was transferred to a large conical flask and a solution of methanol $(15 \mathrm{~mL})$ in THF $(50 \mathrm{~mL})$ was added with extreme caution. The mixture was left to stir at $\mathrm{rt}$ for 30 mins. Methanol $(20 \mathrm{~mL})$ was added and the reaction mixture diluted with ethyl acetate and the organic layers washed with water, brine, dried over sodium sulfate and the solvent removed in vacuo to give upon 
purification by column chromatography (4:1 hexane:ethyl acetate) $\mathbf{4}$ as an anomeric mixture as a colourless oil (18.8 g, 80\%).

$\mathrm{R}_{\mathrm{f}}\left(9: 1\right.$ hexane:ethyl acetate, E') 0.26; $v_{\max }($ neat $) / \mathrm{cm}^{-1} 3496$ (bw), $2943(\mathrm{~m}), 2868(\mathrm{~m})$, $1464(\mathrm{~m}), 1385(\mathrm{w}), 1336(\mathrm{w}), 1248(\mathrm{w}), 1227(\mathrm{w}), 1134(\mathrm{w}), 1080(\mathrm{~m}), 1028(\mathrm{~s}), 1003$ (m), $885(\mathrm{~m}), 693(\mathrm{~m}) ; \delta_{\mathrm{H}}\left(400 \mathrm{MHz}, \mathrm{CDCl}_{3}\right)$ major anomer: $5.00\left(1 \mathrm{H}, \mathrm{s}, \mathbf{H}^{1}{ }^{\prime}\right), 4.53(1 \mathrm{H}$, $\left.\mathrm{dd}, J=7.5,4.5 \mathrm{~Hz}, \mathbf{H}^{3^{\prime}}\right), 3.78-4.00\left(6 \mathrm{H}, \mathrm{m}, \mathbf{H}^{2^{\prime}}, \mathbf{H}^{4^{\prime}}, \mathbf{H}^{1}, \mathbf{H}^{2}\right), 3.69-3.74\left(2 \mathrm{H}, \mathrm{m}, \mathbf{H}^{5^{\prime}}\right)$, $2.83(1 \mathrm{H}, \mathrm{bs}, s \mathrm{Bu}-\mathrm{CH}), 1.73\left(1 \mathrm{H}, \mathrm{bs}, \mathrm{OH}^{2}\right), 1.38-1.55\left(2 \mathrm{H}, \mathrm{m}, s \mathrm{Bu}-\mathrm{CH}_{2}\right), 1.07-1.16$ (28H, m, TIPDS), 0.85-0.92 (6H, m, sBu-CH$) ; \delta_{\mathrm{C}}\left(100 \mathrm{MHz}, \mathrm{CDCl}_{3}\right)$ major anomer: $102.6\left(\mathrm{~d}, \mathbf{C}^{1}\right), 84.6\left(\mathrm{~d}, \mathbf{C}^{2}\right), 84.3\left(\mathrm{~d}, \mathbf{C}^{4^{\prime}}\right), 73.3\left(\mathrm{~d}, \mathbf{C}^{3}\right), 73.3(\mathrm{~d}, s \mathrm{Bu}-\mathbf{C H}), 70.8\left(\mathrm{t}, \mathbf{C}^{1}\right)$,

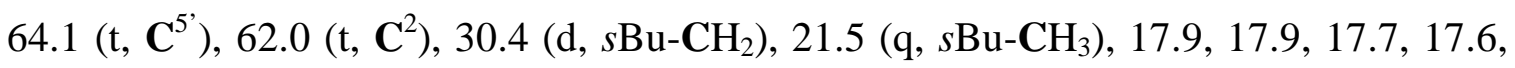
17.5, 17.4, 17.3, 17.2 (q, TIPDS-CH 3 ), 13.6, 13.5, 13.4, 13.0 (d, TIPDS-CH), 10.2 (q, $\left.s \mathrm{Bu}-\mathrm{CH}_{3}\right) ; m / z$ LRMS [ES $\left.{ }^{+}, \mathrm{MeOH}\right] 515\left(\mathrm{M}+\mathrm{Na}^{+}, 100 \%\right) ; m / z$ HRMS [ES $\left.{ }^{+}, \mathrm{MeOH}\right]$ found 515.2841 $\left(\mathrm{M}+\mathrm{Na}^{+}\right) \mathrm{C}_{23} \mathrm{H}_{48} \mathrm{O}_{7} \mathrm{Si}_{2} \mathrm{Na}$ requires 515.2831.

\section{Isobutyl-2'-O-(2-phthalimidoethyl)-3',5'-O-(1,1,3,3,-tetraisopropyl-disiloxane-1,3-} diyl)-D-ribose, 5

Compound 4 (18.8 g, $38.1 \mathrm{mmol})$ was dissolved in THF (100 mL). Triphenylphosphine (11.0 g, $41.9 \mathrm{mmol})$ and phthalimide $(6.18 \mathrm{~g}, 41.9 \mathrm{mmol})$ were added and the resulting suspension stirred at $0{ }^{\circ} \mathrm{C}$ under an argon atmosphere before adding DEAD (6.60 mL, $41.9 \mathrm{mmol})$ as a solution in THF $(40 \mathrm{~mL})$. The reaction mixture was left to warm to rt and stirred for $2 \mathrm{~h}$. The solvent was removed in vacuo and the crude product purified by 
column chromatography (19:1 hexane:ethyl acetate then 4:1 hexane:ethyl acetate) to give 5 as an anomeric mixture as a yellow solid (17.4 $\mathrm{g}, 73 \%)$.

$\mathrm{R}_{\mathrm{f}}\left(4: 1\right.$ hexane:ethyl acetate, E') 0.21; $v_{\max }($ neat $) / \mathrm{cm}^{-1} 3198(\mathrm{w}), 3063(\mathrm{~m}), 2941(\mathrm{~m})$, $2868(\mathrm{~m}), 1724(\mathrm{~m}), 1604(\mathrm{w}), 1465(\mathrm{~m}), 1383(\mathrm{~m}), 1306(\mathrm{~m}), 1135(\mathrm{~m}), 1034(\mathrm{~s}), 884$ $(\mathrm{m}), 710(\mathrm{~m}) ; \delta_{\mathrm{H}}\left(400 \mathrm{MHz}, \mathrm{CD}_{3} \mathrm{OD}\right)$ major anomer: 7.82-7.90 (4H, m, Phth-CH $\left.{ }^{\mathrm{Ar}}\right), 5.08$ $\left(1 \mathrm{H}, \mathrm{s}, \mathbf{H}^{1}\right), 4.58\left(1 \mathrm{H}, \mathrm{dd}, J=7.5,4.0 \mathrm{~Hz}, \mathbf{H}^{3 \prime}\right), 3.95-4.03\left(3 \mathrm{H}, \mathrm{m}, \mathbf{H}^{2}{ }^{\prime}\right.$ plus $\left.\mathbf{H}^{2}\right), 3.80(1 \mathrm{H}$, $\left.\mathrm{dd}, J=8.0,4.0 \mathrm{~Hz}, \mathbf{H}^{4}\right), 3.69-3.76\left(4 \mathrm{H}, \mathrm{m}, \mathbf{H}^{5^{\prime}}\right.$ plus $\left.\mathbf{H}^{1}\right), 3.36(1 \mathrm{H}, \mathrm{bs}, s \mathrm{Bu}-\mathrm{CH}), 1.46-$ $1.60\left(2 \mathrm{H}, \mathrm{m}, s \mathrm{Bu}-\mathrm{CH}_{2}\right), 1.10-1.17(28 \mathrm{H}, \mathrm{m}, \mathrm{TIPDS}), 0.93-1.00\left(6 \mathrm{H}, \mathrm{m}, s \mathrm{Bu}-\mathrm{CH}_{3}\right) ; \delta_{\mathrm{C}}$ (100 MHz, $\left.\mathrm{CD}_{3} \mathrm{OD}\right)$ major anomer: $170.9(\mathrm{~s}, \mathbf{C O}), 135.3\left(\mathrm{~d}, \mathbf{C H}^{\mathrm{Ar}}\right), 134.3,132.9\left(\mathrm{~s}, \mathbf{C}^{3}\right)$, $105.7\left(\mathrm{~d}, \mathbf{C}^{1}\right), 85.5\left(\mathrm{~d}, \mathbf{C}^{2}\right), 85.2\left(\mathrm{~d}, \mathbf{C}^{4^{\prime}}\right), 74.8\left(\mathrm{~d}, \mathbf{C}^{3}\right), 74.2(\mathrm{~d}, s \mathrm{Bu}-\mathbf{C H}), 65.3\left(\mathrm{t}, \mathbf{C}^{1}\right)$, $62.5\left(\mathrm{t}, \mathbf{C}^{5}\right), 31.1\left(\mathrm{t}, \mathbf{C}^{2}\right), 30.0\left(\mathrm{t}, s \mathrm{Bu}-\mathrm{CH}_{2}\right), 21.4\left(\mathrm{t}, s \mathrm{Bu}-\mathrm{CH}_{3}\right)$, 18.0, 17.9, 17.8, 17.7, 17.7, 17.5, 17.5, 17.4 (q, TIPDS- $\left.\mathrm{CH}_{3}\right), 14.2,14.1,14.1,14.0$ (d, TIPDS-CH), 10.3 (q, $\left.s \mathrm{Bu}-\mathrm{CH}_{3}\right) ; \mathrm{m} / z$ LRMS [ES, $\left.\mathrm{MeOH}\right] 644\left(\mathrm{M}+\mathrm{Na}^{+}, 100 \%\right) ; \mathrm{m} / z \mathrm{HRMS}\left[\mathrm{ES}^{+}, \mathrm{MeOH}\right]$ found $644.3051\left(\mathrm{M}+\mathrm{Na}^{+}\right) \mathrm{C}_{31} \mathrm{H}_{51} \mathrm{O}_{8} \mathrm{NSi}_{2} \mathrm{Na}$ requires 644.3045 .

\section{2'-O-(2-Phthalimidoethyl)-3',5'-O-(1,1,3,3,-tetraisopropyldisiloxane-1,3-diyl)-D-}

\section{ribofuranose, 6}

Compound 5 (15.8 g, $25.4 \mathrm{mmol})$ was dissolved in DCM (400 mL) and cooled to $15{ }^{\circ} \mathrm{C}$ (ice/methanol) before adding TFA $(25 \mathrm{~mL})$ followed by water $(1.4 \mathrm{~mL})$. The reaction mixture was left to stir at $-15{ }^{\circ} \mathrm{C}$ for $3 \mathrm{~h}$. Triethylamine $(45 \mathrm{~mL})$ was added cautiously and the reaction mixture washed with saturated sodium bicarbonate solution (2 x $150 \mathrm{~mL}$ ). The organic layer was separated and dried over sodium sulfate and the 
solvent removed in vacuo. The crude product was purified by column chromatography (1:1 hexane:ethyl acetate) to give 6 as a pale yellow oil $(12.0 \mathrm{~g}, 83 \%)$.

$\mathrm{R}_{\mathrm{f}}\left(1: 1\right.$ hexane:ethyl acetate, B') 0.56; $v_{\max }($ neat $) / \mathrm{cm}^{-1} 3470(\mathrm{bw}), 2944(\mathrm{~m}), 2867(\mathrm{~m})$, $1774(w), 1711(\mathrm{~s}), 1466(\mathrm{w}), 1428(\mathrm{w}), 1394(\mathrm{~s}), 1366(\mathrm{w}), 1248(\mathrm{w}), 1128(\mathrm{w}), 1038(\mathrm{~s})$, $885(\mathrm{~m}), 720(\mathrm{~m}) ; \delta_{\mathrm{H}}\left(400 \mathrm{MHz}, \mathrm{CDCl}_{3}\right)$ major isomer: $7.85(2 \mathrm{H}, \mathrm{dd}, J=5.5,3.0 \mathrm{~Hz}$, $\left.\mathbf{H}^{4}\right), 7.71\left(2 \mathrm{H}, \mathrm{dd}, J=5.5,3.0 \mathrm{~Hz}, \mathbf{H}^{5}\right), 5.29\left(1 \mathrm{H}, \mathrm{dd}, J=11.5,4.0 \mathrm{~Hz}, \mathbf{H}^{1}\right), 4.38(1 \mathrm{H}$, app.p, $\left.J=5.0 \mathrm{~Hz}, \mathbf{H}^{3}\right), 4.20\left(1 \mathrm{H}, \mathrm{dd}, J=9.0,4.5 \mathrm{~Hz}, \mathbf{H}^{4}\right), 3.73-3.97\left(7 \mathrm{H}, \mathrm{m}, \mathbf{H}^{{ }^{\prime}}, \mathbf{H}^{{ }^{\prime}}, \mathbf{H}^{1}\right.$ plus $\left.\mathbf{H}^{2}\right), 1.02-1.07(28 \mathrm{H}, \mathrm{m}, \mathrm{TIPDS}) ; \delta_{\mathrm{C}}\left(100 \mathrm{MHz}, \mathrm{CDCl}_{3}\right)$ major isomer: 168.9 (s, CO), 134.8, $134.6\left(\mathrm{~d}, \mathbf{C}^{5}\right), 132.6,132.5\left(\mathrm{~s}, \mathbf{C}^{3}\right), 124.1,123.8\left(\mathrm{~d}, \mathbf{C}^{4}\right), 95.8\left(\mathrm{~d}, \mathbf{C}^{1}\right), 79.8$ $\left(\mathrm{d}, \mathbf{C}^{2}\right), 79.6\left(\mathrm{~d}, \mathbf{C}^{4}\right), 72.6\left(\mathrm{~d}, \mathbf{C}^{3}\right), 70.8\left(\mathrm{t}, \mathbf{C}^{5^{\prime}}\right), 60.9\left(\mathrm{t}, \mathbf{C}^{1}\right), 38.8\left(\mathrm{t} . \mathbf{C}^{2}\right), 17.9,17.8$, 17.7, 17.6, 17.6, 17.5, 17.4, 17.4 (q, TIPDS-CH 3$), 14.0,13.9,13.6,13.4$ (d, TIPDS-CH); $m / z$ LRMS $\left[\mathrm{ES}^{+}, \mathrm{MeOH}\right] 588\left(\mathrm{M}+\mathrm{Na}^{+}, 100 \%\right) ; m / z \mathrm{HRMS}\left[\mathrm{ES}^{+}, \mathrm{MeOH}\right]$ found 588.2439 $\left(\mathrm{M}+\mathrm{Na}^{+}\right) \mathrm{C}_{27} \mathrm{H}_{43} \mathrm{NO}_{8} \mathrm{Si}_{2} \mathrm{Na}$ requires 588.2419.

\section{Ethyl[2'-O-ethylphthalimido-3',5'-O-(1,1,3,3,-tetraisopropyldisiloxane-1,3-diyl)- $\beta$-D- erythro-pentofuranosyl]acetate, 8}

Compound 6 (2.5 g, $4.29 \mathrm{mmol})$ was dissolved in dry THF (55 ml) and ethoxycarbonyl(methylene)triphenyl phosphorane $(1.79 \mathrm{~g}, 5.15 \mathrm{mmol})$ was added. The reaction mixture was stirred at $50{ }^{\circ} \mathrm{C}$ for $2 \mathrm{~h}$ then evaporated to dryness. The residue was redissolved in ethanol and sodium ethoxide $(20 \mathrm{mg}, 0.29 \mathrm{mmol})$ was added. The reaction mixture was stirred at reflux for $2 \mathrm{~h}$ after which the solvent was removed in vacuo. 
Purification by column chromatography (8.5:1.5 hexane:ethyl acetate) afforded $\mathbf{8}$, a separable anomeric mixture, as a pale yellow foam $\left(\mathbf{8}_{\alpha}: \mathbf{8}_{\beta}, 0.9: 1,51 \%\right)$.

$\alpha$-anomer:

$\mathrm{R}_{\mathrm{f}}\left(1: 1\right.$ hexane:ethyl acetate, PMA) $0.27 ; \delta_{\mathrm{H}}\left(400 \mathrm{MHz}, \mathrm{CDCl}_{3}\right) 7.84(2 \mathrm{H}, \mathrm{dd}, J=5.5,3.0$ $\mathrm{Hz}$, Phth-CH$\left.{ }^{\mathrm{Ar}}\right), 7.70\left(2 \mathrm{H}, \mathrm{dd}, J=5.0,3.0 \mathrm{~Hz}\right.$, Phth-CH$\left.{ }^{\mathrm{Ar}}\right), 4.40(1 \mathrm{H}, \mathrm{ddd}, J=9.0,6.0$, $\left.4.0 \mathrm{~Hz}, \mathbf{H}^{1}\right), 4.30\left(1 \mathrm{H}, \mathrm{dd}, J=9.5,4.0 \mathrm{~Hz}, \mathbf{H}^{3 ’}\right), 4.22(1 \mathrm{H}, \mathrm{ddd}, J=9.5,6.0,5.5 \mathrm{~Hz}$, $\mathrm{NCH}), 4.01(1 \mathrm{H}, \mathrm{ddd}, J=14.0,7.0,4.0 \mathrm{~Hz}, \mathrm{NCH}), 3.66-4.04\left(7 \mathrm{H}, \mathrm{m}, \mathrm{OCH}, \mathrm{COCH}_{2}, \mathbf{H}^{2}\right.$, $\left.\mathbf{H}^{4},{ }^{5} \mathrm{CH}_{2}\right), 3.69(1 \mathrm{H}, \mathrm{ddd}, J=11.6,6.5,4.5 \mathrm{~Hz}, \mathrm{OCH}) 2.69(1 \mathrm{H}, \mathrm{dd}, J=16.6,9.0 \mathrm{~Hz}$, CHCO), $2.51(1 \mathrm{H}, \mathrm{dd}, J=16.6,6.0 \mathrm{~Hz}, \mathrm{CHCO}), 1.17\left(3 \mathrm{H}, \mathrm{t}, J=7.0 \mathrm{~Hz}, \mathrm{CH}_{2} \mathrm{CH}_{3}\right), 0.93-$ $1.06(28 \mathrm{H}, \mathrm{m}, \mathrm{TIPDS}) ; \delta_{\mathrm{C}}\left(100 \mathrm{MHz}, \mathrm{CDCl}_{3}\right) 169.8$ (s, $\left.\mathrm{CH}_{2} \mathrm{CO}\right), 166.6(\mathrm{~s}, \mathrm{NCO}), 132.3$ $\left(\mathrm{d}, \mathbf{C}^{5}\right), 130.7\left(\mathrm{~s}, \mathbf{C}^{3}\right), 121.7\left(\mathrm{~d}, \mathbf{C}^{4}\right), 82.30\left(\mathrm{~d}, \mathbf{C}^{2}\right), 80.8\left(\mathrm{~d}, \mathbf{C}^{4^{\prime}}\right), 79.8\left(\mathrm{~d}, \mathbf{C}^{1}\right), 71.7(\mathrm{~d}$ $\left.\mathbf{C}^{3}\right), 68.0\left(\mathrm{t}, \mathrm{OCH}_{2} \mathrm{CH}_{2}\right), 60.8\left(\mathrm{t}, \mathbf{C}^{5}\right), 60.6\left(\mathrm{t}, \mathrm{OCH}_{2} \mathrm{CH}_{3}\right), 39.3\left(\mathrm{t}, \mathrm{CH}_{2} \mathrm{~N}\right), 38.3(\mathrm{t}$, $\mathbf{C H}_{2} \mathrm{CO}$ ), 17.70, 17.44, 17.32, (q, TIPDS- $\left.\mathrm{CH}_{3}\right), 14.6\left(\mathrm{q}, \mathrm{CH}_{2} \mathbf{C H}_{3}\right), 12.7,12.0,11.6,11.2$ (d, TIPDS-CH); $m / z$ LRMS $\left[\mathrm{ES}^{+}, \mathrm{MeOH}\right] 636.4\left(\mathrm{M}+\mathrm{H}^{+}, 100 \%\right), 658.6\left(\mathrm{M}+\mathrm{Na}^{+}, 50 \%\right)$; $m / z$ HRMS [ES $\left.{ }^{+}, \mathrm{MeOH}\right]$ found $658.2843 \mathrm{C}_{31} \mathrm{H}_{49} \mathrm{NO}_{9} \mathrm{Si}_{2} \mathrm{Na}$ requires 658.2838 .

\section{$\beta$-anomer:}

$\mathrm{R}_{\mathrm{f}}\left(1: 1\right.$ hexane:ethyl acetate, PMA) $0.25 ; \delta_{\mathrm{H}}\left(400 \mathrm{MHz}, \mathrm{CDCl}_{3}\right) 7.83(2 \mathrm{H}, \mathrm{dd}, J=5.5,3.0$ $\left.\mathrm{Hz}, \mathbf{H}^{4}\right), 7.70\left(2 \mathrm{H}, \mathrm{dd}, J=5.0,3.0 \mathrm{~Hz}, \mathbf{H}^{5}\right), 4.18 \sim 4.12\left(2 \mathrm{H}, \mathrm{m}, \mathbf{H}^{1}, \mathbf{H}^{3 \prime}\right), 4.06(2 \mathrm{H}, \mathrm{q}, J=$ 7.0 Hz, $\left.\mathrm{OCH}_{2} \mathrm{CH}_{3}\right), 3.72-3.96\left(8 \mathrm{H}, \mathrm{m} \mathrm{CH}_{2} \mathrm{~N}, \mathrm{OCH}_{2} \mathrm{CH}_{2}, \mathbf{H}^{2}, \mathbf{H}^{4},{ }^{5} \mathrm{CH}_{2}\right), 2.58(1 \mathrm{H}, \mathrm{dd}, J$ $=16.0,6.0 \mathrm{~Hz}, \mathrm{CHCO}), 2.45(1 \mathrm{H}, \mathrm{dd}, J=16.0,7.0 \mathrm{~Hz}, \mathrm{CHCO}), 1.21(3 \mathrm{H}, \mathrm{t}, J=7.0 \mathrm{~Hz}$, $\left.\mathrm{OCH}_{2} \mathrm{CH}_{3}\right), 0.93-1.06(28 \mathrm{H}, \mathrm{m}, \mathrm{TIPDS}) ; \delta_{\mathrm{C}}\left(100 \mathrm{MHz}, \mathrm{CDCl}_{3}\right) 170.7\left(\mathrm{~s}, \mathrm{CH}_{2} \mathrm{CO}\right), 168.6$ (s, NCO), $134.1\left(\mathrm{~d}, \mathbf{C}^{5}\right), 132.6\left(\mathrm{~s}, \mathbf{C}^{3}\right), 123.6\left(\mathrm{~d}, \mathbf{C}^{4}\right), 82.4\left(\mathrm{~d}, \mathbf{C}^{2}\right), 80.9\left(\mathrm{~d}, \mathbf{C}^{4}\right), 79.9(\mathrm{~d}$, 
$\left.\mathbf{C}^{1^{\prime}}\right), 71.8\left(\mathrm{~d}, \mathbf{C}^{3^{\prime}}\right), 68.1\left(\mathrm{t}, \mathrm{OCH}_{2} \mathrm{CH}_{2}\right), 60.9\left(\mathrm{t}, \mathbf{C}^{5^{\prime}}\right), 60.8\left(\mathrm{t}, \mathrm{OCH}_{2} \mathrm{CH}_{3}\right), 39.4\left(\mathrm{t}, \mathbf{C H}_{2} \mathrm{CO}\right)$, $38.4\left(\mathrm{t}, \mathrm{CH}_{2} \mathrm{~N}\right), 17.8,17.7,17.6,17.5,17.3\left(\mathrm{q}\right.$, TIPDS- $\left.\mathrm{CH}_{3}\right), 14.5\left(\mathrm{q}, \mathrm{CH}_{2} \mathbf{C H}_{3}\right), 13.8$, 13.5, 13.1 (d, TIPDS-CH); m/z LRMS [ES $\left.{ }^{+}, \mathrm{MeOH}\right] 658.5\left(\mathrm{M}+\mathrm{Na}^{+}, 100 \%\right) ; m / z$ HRMS $\left[\mathrm{ES}^{+}, \mathrm{MeOH}\right]$ found $658.2853 \mathrm{C}_{31} \mathrm{H}_{49} \mathrm{NO}_{9} \mathrm{Si}_{2} \mathrm{Na}$ requires 658.2838 .

\section{2'-O-(Ethylphthalimido)-5'-O-[(4,4'-dimethoxyltrityl)- $\beta$-D-erythro-} pentofuranosyl]acetic acid, 10

Compound $8_{\beta}$ (707 mg, $1.11 \mathrm{mmol}$ ) was dissolved in a mixture of THF (35 ml), conc. $\mathrm{HCl}(35 \mathrm{ml})$ and distilled water $(10 \mathrm{ml})$ then stirred at reflux for $5 \mathrm{~h}$. The solvent was then carefully removed in vacuo and the residue was co-evaporated with anhydrous pyridine $(2 \times 50 \mathrm{ml})$ then redissolved in anhydrous pyridine $(50 \mathrm{ml})$. A solution of 4,4'-

dimethoxytrityl chloride $(0.38 \mathrm{~g}, 1.13 \mathrm{mmol})$ in pyridine $(20 \mathrm{ml})$ was added dropwise and the reaction mixture was stirred at $\mathrm{rt}$ for $5 \mathrm{~h}$, then quenched with methanol $(50 \mathrm{ml})$. The solvent was removed in vacuo to afford $\mathbf{1 0}$ as a yellow solid after purification by column chromatography (1:4 hexane:ethyl acetate).

$\mathrm{R}_{\mathrm{f}}\left(1: 4\right.$ hexane:ethyl acetate, PMA) $0.23 ; \delta_{\mathrm{H}}\left(400 \mathrm{MHz}, \mathrm{CDCl}_{3}\right) 8.57(1 \mathrm{H}, \mathrm{bs}, \mathrm{COOH})$, $7.77\left(2 \mathrm{H}, \mathrm{dd}, J=5.5,3.0 \mathrm{~Hz}, \mathbf{H}^{4}\right), 7.64\left(2 \mathrm{H}, \mathrm{dd}, J=5.0,3.0 \mathrm{~Hz}, \mathbf{H}^{5}\right), 7.09-7.35(9 \mathrm{H}, \mathrm{m}$, $\left.\mathrm{CH}^{\mathrm{Ar}}\right), 6.74\left(4 \mathrm{H}, \mathrm{d}, J=9.0 \mathrm{~Hz}, \mathrm{CH}^{\mathrm{Ar}}\right), 4.20\left(1 \mathrm{H}\right.$, app. q, $\left.J=5.9 \mathrm{~Hz}, \mathbf{H}^{1}{ }^{\prime}\right), 3.85-3.89(4 \mathrm{H}$, $\left.\mathrm{m}, \mathbf{H}^{3^{\prime}}, \mathbf{H}^{4}, \mathrm{CH}_{2} \mathrm{~N}\right), 3.66-3.75\left(3 \mathrm{H}, \mathrm{m}, \mathbf{H}^{2}, \mathrm{OCH}_{2} \mathrm{CH}_{3}\right), 3.70\left(6 \mathrm{H}, \mathrm{s}, \mathrm{OCH}_{3}\right), 3.23(1 \mathrm{H}$, $\left.\mathrm{dd}, J=10.5,3.0 \mathrm{~Hz}, \mathbf{H}^{5^{\prime}}\right), 3.04\left(1 \mathrm{H}, \mathrm{dd}, J=10.5,4.0 \mathrm{~Hz}, \mathbf{H}^{5^{\prime}}\right), 2.62(1 \mathrm{H}, \mathrm{dd}, J=15.6$, $7.0 \mathrm{~Hz}, \mathrm{CHCO}), 2.45(1 \mathrm{H}, \mathrm{dd}, J=15.6,6.0 \mathrm{~Hz}, \mathrm{CHCO}) ; \delta_{\mathrm{C}}\left(100 \mathrm{MHz}, \mathrm{CDCl}_{3}\right) 168.9$ (s, $\left.\mathrm{CH}_{2} \mathbf{C O}\right), 158.8$ (s, NCO), $134.6\left(\mathrm{~d}, \mathbf{C}^{5}\right), 132.3\left(\mathrm{~s}, \mathbf{C}^{3}\right), 123.9\left(\mathrm{~d}, \mathbf{C}^{4}\right), 145.3,136.5,130.5$, 
129.4, 128.6, 128.2, 127.1, 125.7, $113.5\left(\mathrm{~d}, \mathbf{C} \mathrm{H}^{\mathrm{Ar}}\right), 86.5\left(\mathrm{q}, \mathbf{C}^{\mathrm{Ar}}\right), 83.8\left(\mathrm{~d}, \mathbf{C}^{2}\right), 83.6(\mathrm{~d}$, $\left.\mathbf{C}^{4^{\prime}}\right), 77.2\left(\mathrm{~d}, \mathbf{C}^{1^{\prime}}\right), 71.3\left(\mathrm{~d}, \mathbf{C}^{3}\right), 68.7\left(\mathrm{t}, \mathrm{OCH}_{2} \mathrm{CH}_{3}\right), 64.1\left(\mathrm{t}, \mathbf{C}^{5^{\prime}}\right), 55.6\left(\mathrm{q}, \mathrm{OCH}_{3}\right), 38.8(\mathrm{t}$, $\left.\mathrm{CH}_{2} \mathrm{CO}\right), 38.4\left(\mathrm{t}, \mathrm{CH}_{2} \mathrm{~N}\right) ; \mathrm{m} / z$ LRMS [ES $\left.{ }^{+}, \mathrm{CH}_{3} \mathrm{CN}\right] 690\left(\mathrm{M}+\mathrm{Na}^{+}, 100 \%\right) ; \mathrm{m} / z \mathrm{HRMS}$ $\left[\mathrm{ES}^{+}, \mathrm{CH}_{3} \mathrm{CN}\right]$ found $690.2309 \mathrm{C}_{38} \mathrm{H}_{37} \mathrm{NO}_{10} \mathrm{Na}$ requires 690.2296 .

\section{$N$-[3-(4-Acetamidothiazol-2-yl)phenyl]-[2'-O-ethylphthalimido-5'-O-(4,4'- dimethoxytrityl)- $\beta$-D-erythro-pentofuranosyl]acetamide, 11}

Compound 10 (0.24 g, $0.35 \mathrm{mmol})$ and $N$-(2-(3-aminophenyl)thiazol-4-yl)acetamide (94 $\mathrm{mg}, 0.42 \mathrm{mmol}$ ) (reference 28) were dissolved in DCM (20 ml) and dicyclohexylcarbodiimide ( $87 \mathrm{mg}, 0.42 \mathrm{mmol}), n$-butanol $(9 \mathrm{ml})$ and pyridine $(4 \mathrm{ml})$ were added. The reaction was stirred at $\mathrm{rt}$ for $4 \mathrm{~h}$ after which the solvent was removed in vacuo. The residue which was purified by column chromatography (1:1 ethyl acetate:diethyl ether) to afford $\mathbf{1 1}$ as a foam $(247 \mathrm{mg}, 80 \%)$.

$\mathrm{R}_{\mathrm{f}}\left(1: 1\right.$, acetate:diethyl ether, PMA) $0.21 ; \delta_{\mathrm{H}}\left(400 \mathrm{MHz}, \mathrm{CDCl}_{3}\right) 10.01(1 \mathrm{H}, \mathrm{bs}, \mathrm{CONH})$, $8.20(1 \mathrm{H}, \mathrm{s}, \mathrm{CONH}), 7.74\left(2 \mathrm{H}, \mathrm{dd}, J=5.5,3.0 \mathrm{~Hz}, \mathbf{H}^{4}\right), 7.61(2 \mathrm{H}, \mathrm{dd}, J=5.0,3.0 \mathrm{~Hz}$, $\left.\mathbf{H}^{5}\right)$; 7.09-7.40 (14H, m, CH$\left.{ }^{\mathrm{Ar}}\right), 6.68\left(4 \mathrm{H}, \mathrm{d}, J=8.0 \mathrm{~Hz}, \mathrm{C} \mathbf{H}^{\mathrm{Ar}}\right), 4.21(1 \mathrm{H}, \mathrm{dd}, J=15.0$, $\left.6.8 \mathrm{~Hz}, \mathbf{H}^{1^{\prime}}\right), 4.18-4.25\left(2 \mathrm{H}, \mathrm{m}, \mathrm{OH}^{3^{\prime}}, \mathbf{H}^{3^{\prime}}\right), 3.90-3.92\left(3 \mathrm{H}, \mathrm{m}, \mathbf{H}^{4^{\prime}}, \mathrm{CH}_{2} \mathrm{~N}\right), 364-3.73(3 \mathrm{H}$, m, $\left.\mathbf{H}^{2}, \mathrm{OCH}_{2} \mathrm{CH}_{2}\right), 3.64\left(3 \mathrm{H}, \mathrm{s}, \mathrm{OCH}_{3}\right), 3.63\left(3 \mathrm{H}, \mathrm{s}, \mathrm{OCH}_{3}\right), 3.42(1 \mathrm{H}, \mathrm{dd}, J=10.5,2.5$ $\left.\mathrm{Hz}, \mathbf{H}^{5^{\prime}}\right), 3.29\left(1 \mathrm{H}, \mathrm{dd}, J=10.5,4.5 \mathrm{~Hz}, \mathbf{H}^{5 \prime}\right), 2.61\left(2 \mathrm{H}, \mathrm{d}, J=6.5 \mathrm{~Hz}, \mathrm{CH}_{2} \mathrm{CO}\right), 2.04(3 \mathrm{H}$, s, $\left.\mathrm{COCH}_{3}\right) ; \delta_{\mathrm{C}}\left(100 \mathrm{MHz}, \mathrm{CDCl}_{3}\right) 168.9\left(\mathrm{~s}, \mathrm{CH}_{2} \mathrm{CO}\right), 168.5\left(\mathrm{~s}, \mathrm{NHCOCH}_{3}\right), 158.9(\mathrm{~s}$, NCO), 158.3 (s, NCO), $134.6\left(\mathrm{~d}, \mathbf{C}^{5}\right), 132.3\left(\mathrm{~s}, \mathbf{C}^{3}\right), 123.9\left(\mathrm{~d}, \mathbf{C}^{4}\right), 150.2,149.5,145.2$, 138.6, 136.4, 136.2, 130.5, 130.4, 129.6, 128.6, 128.3, 127.3, 124.1, 122.1, 119.9, 118.2, 
113.6, $108.4\left(\mathrm{~d}, \mathbf{C} \mathrm{H}^{\mathrm{Ar}}\right), 86.7\left(\mathrm{~s}, \mathbf{C}^{\mathrm{Ar}}\right), 84.3\left(\mathrm{~d}, \mathbf{C}^{2^{\prime}}\right), 83.7\left(\mathrm{~d}, \mathbf{C}^{4^{\prime}}\right), 77.8\left(\mathrm{~d}, \mathbf{C}^{1^{\prime}}\right), 71.3(\mathrm{~d}$, $\left.\mathrm{C}^{3^{\prime}}\right), 69.0\left(\mathrm{t}, \mathrm{OCH}_{2} \mathrm{CH}_{3}\right), 64.0\left(\mathrm{t}, \mathbf{C}^{5^{\prime}}\right), 55.6\left(\mathrm{q}, \mathrm{OCH}_{3}\right), 42.4\left(\mathrm{t}, \mathbf{C H}_{2} \mathrm{CO}\right), 38.4\left(\mathrm{t}, \mathbf{C H}_{2} \mathrm{~N}\right)$, $23.4\left(\mathrm{q}, \mathrm{COCH}_{3}\right) ; \mathrm{m} / z \mathrm{LRMS}\left[\mathrm{ES}^{+}, \mathrm{CH}_{3} \mathrm{CN}\right] 905\left(\mathrm{M}+\mathrm{Na}^{+}, 100 \%\right) ; \mathrm{m} / z \mathrm{HRMS}^{2}\left[\mathrm{ES}^{+}\right.$, $\left.\mathrm{CH}_{3} \mathrm{CN}\right]$ found $905.2826 \mathrm{C}_{49} \mathrm{H}_{46} \mathrm{~N}_{6} \mathrm{O}_{10} \mathrm{SNa}$ requires 905.2827.

\section{$N$-[3-(4-acetamidothiazol-2-yl)phenyl]-[2'-O-ethylphthalimido-5'-O-(4,4'-} dimethoxyltrityl)]-3'-O-(2-cyanoethyl-diisopropylphosphoramidyl)- $\beta$-D-erythropentofuranosyl)acetamide, 12

Compound 11 (0.247 g, $0.28 \mathrm{mmol})$ was dissolved in anhydrous THF and DIPEA (0.315 $\mathrm{ml}, 0.84 \mathrm{mmol})$ and 2-cyanoethyl $N, N$-diisopropyl chlorophosphine $(0.074 \mathrm{ml}, 0.336$ mmol) were added. The reaction mixture was stirred at $\mathrm{rt}$ for $5 \mathrm{~h}$ then diluted with ethyl acetate and washed with saturated $\mathrm{KCl}$. The organic layer was dried over sodium sulphate, evaporated to dryness, and the crude product was purified by column chromatography (1:1 ethyl acetate:toluene) to afford $\mathbf{1 2}$ as a pale yellow oil (253 $\mathrm{mg}$, $84 \%)$.

$\mathrm{R}_{\mathrm{f}}\left(2: 3\right.$ ethyl acetate:toluene, PMA) $0.21 ; \mathrm{m} / z$ LRMS $\left[\mathrm{ES}^{+}, \mathrm{CH}_{3} \mathrm{CN}\right] 1105\left(\mathrm{M}+\mathrm{Na}^{+}\right.$, $100 \%) ; \delta_{\mathrm{P}}\left(121 \mathrm{MHz}, \mathrm{CDCl}_{3}\right)$ 151.1, 149.3.

\section{Phosphoramidite drying}

The special phosphoramidites synthesised for this study were treated as follows: After purification by column chromatography each monomer was dried under high vacuum overnight, dissolved in anhydrous acetonitrile and filtered through a Millipore Millex®- 
FH syringe filter $(0.45 \mu \mathrm{m}, 25 \mathrm{~mm})$. The solvent was then removed and the monomer was redissolved in anhydrous DCM. Aliquots of $100 \mu \mathrm{L}$ corresponding to $100 \mu$ moles were transferred to ABI-style monomer bottles and dried in a desiccator overnight under high vaccum before being stored under slight positive pressure of argon at $-20^{\circ} \mathrm{C}$.

\section{Preparation of synthetic oligonucleotides}

All oligonucleotides were synthesised on an Applied Biosystems 394 automated DNA/RNA synthesiser using the standard 0.2 mmole phosphoramidite cycle of acidcatalysed detritylation, coupling, capping and iodine oxidation. Stepwise coupling efficiencies and overall yields were determined by the automated trityl cation conductivity monitoring facility and in all cases were $>98.0 \%$. All $\beta$-Cyanoethyl phosphoramidite monomers were dissolved in anhydrous acetonitrile to a concentration of $0.1 \mathrm{M}$ immediately prior to use. Standard DNA phosphoramidites, solid supports and additional reagents were purchased from Link Technologies Ltd or Applied Biosystems Ltd. Oligonucleotides were cleaved and deprotected at room temperature for $24 \mathrm{~h}$ using 2 $\mathrm{ml}$ of $10 \% \mathrm{MeNH}_{2}$ in water. For oligonucleotides containing $\mathrm{S}$ and ${ }^{2 \mathrm{AE}} \mathrm{S}$ mass spectrometry revealed some loss of acetyl (presumably from the thiazole amino group) on longer incubation. Purification was carried out by reversed phase HPLC on a Gilson system using an ABI Aquapore column (C8), $8 \mathrm{~mm}$ x $250 \mathrm{~mm}$, pore size $300 \AA$ A. The system was controlled by Gilson 7.12 software and the following protocol was used: Run time 30 minutes, flow rate $4 \mathrm{ml}$ per min, binary system, gradient: Time in mins (\% buffer B);0 (0); 3(0); 5(20); $21(100) ; 25(100) ; 27$ (0); 30(0). Elution buffer A $0.1 \mathrm{M}$ ammonium acetate, $\mathrm{pH} 7.0$, buffer $\mathrm{B} 0.1 \mathrm{M}$ ammonium acetate with $35 \%$ acetonitrile $\mathrm{pH}$ 7.0. Elution 
of oligonucleotides was monitored by ultraviolet absorption at $295 \mathrm{~nm}$. After HPLC purification oligonucleotides were desalted using disposable NAP 10 Sephadex columns (Pharmacia), aliquoted into Eppendorf tubes and stored at $-20^{\circ} \mathrm{C}$. Purified oligonucleotides were analysed by MALDI-TOF MS using a ThermoBioAnalysis Dynamo MALDI-TOF mass spectrometer in positive ion mode (35) (Table 1).

Table S1. Positive ion MALDI-TOF of modified oligonucleotides $\left(X={ }^{2 \mathrm{AE}} \mathrm{S}, \mathrm{Y}=\mathrm{S}, \mathrm{Me}\right.$ Red $=$ quencher methyl red threoninol).

\begin{tabular}{|l|c|c|}
\hline Oligonucleotide & Required Mass $\mathrm{M}+\mathrm{H}^{+}$ & Actual Mass $\mathrm{M}+\mathrm{H}^{+}$ \\
\hline 5'-TCTCCTTYTTTCT & 3693.6 & 3693.9 .1 \\
\hline 5'-TCTCTTYTTTCT & 3752.6 & 3752.8 \\
\hline 5'-TCTCTCTTXTCCTCC & 5487.7 & 5487.9 \\
\hline 5'-MeRed TCTCTCTTXTCCTCCTCC & 5906.1 & 5907.0 \\
\hline 5'-MeRed TCTCTCTXTCTXTCCTCC & 6129.4 & 6131.1 \\
\hline 5'-MeRed TCTCTCTTYTCCTCCTCC & 6101.4 & 6101.9 \\
\hline 5'-MeRed TCTCTCTYTCTYTCCTCC & 6010.6 & 6012.0 \\
\hline
\end{tabular}

